\title{
TTR
}

Traduction, terminologie, re?daction

\section{Vor dem Gesetz : la porte du traduire}

\section{Alexis Nouss}

Volume 5, numéro 2, 2e semestre 1992

Kafka pluriel : réécriture et traduction

URI : https://id.erudit.org/iderudit/037129ar

DOI : https://doi.org/10.7202/037129ar

Aller au sommaire du numéro

Éditeur(s)

Association canadienne de traductologie

ISSN

0835-8443 (imprimé)

1708-2188 (numérique)

Découvrir la revue

Citer cet article

Nouss, A. (1992). Vor dem Gesetz : la porte du traduire. TTR, 5(2), 223-243.

https://doi.org/10.7202/037129ar

Tous droits réservés ( TTR: traduction, terminologie, rédaction — Les auteurs, 1992

Ce document est protégé par la loi sur le droit d'auteur. L'utilisation des services d'Érudit (y compris la reproduction) est assujettie à sa politique d'utilisation que vous pouvez consulter en ligne.

https://apropos.erudit.org/fr/usagers/politique-dutilisation/ 


\section{Vor dem Gesetz: la porte du traduire}

\section{Alexis Nouss}

Dans le dernier ouvrage paru de Milan Kundera, l'Immortalité, sur la page récapitulant ses cuvres figurent deux mentions d'un profond intérêt. Après l'indication des six précédents romans de Kundera, ces lignes: «Entre 1985 et 1987 les traductions des ouvrages mentionnés ci-dessus ont été entièrement revues par l'auteur et, dès lors, ont la même valeur d'authenticité que le texte tchèque.» $(1990$, p. 4) Et sous l'indication de l'ouvrage lui-même, l'Immortalité, cette phrase: "La traduction, entièrement revue par l'auteur, a la même valeur d'authenticité que le texte tchèque.» $(1990$, p. 4)

Ces mentions de Kundera introduisent deux problématiques. La première tient évidemment à ce concept d'authenticité que le romancier met en avant comme critère de valorisation des textes en question. C'est habituellement sur la notion de fidélité que s'articulent les réflexions sur la traduction, partageant les auteurs entre ceux qui prônent la fidélité au texte de départ, laissant transparaître son étrangeté sous son nouvel aspect, et ceux qui défendent le respect de la langue et de la littérarité d'arrivée, partisans de la naturalisation en somme. La notion d'authenticité apporte un nouvel éclairage au débat en brisant la relation entre les langues qui semblait objectivement - en réalité esthétiquement nécessaire à l'évaluation. Ce qui répondrait au constant souci de Kafka de miner la domination axiologique de la beauté au profit de la vérité: «Notre art, c'est d'être aveuglé par la vérité; seule est vraie la lumière 
sur le visage grotesque qui recule, rien d'autre.» (tr. M. Robert, 1957, p. 44)

La seconde problématique est précisément contenue dans cette invitation à considérer les deux textes en soi et non dans leur dépendance, poussant à sa limite l'idée qu'une traduction est une écriture et non pas une réécriture, à moins que toute écriture ne le soit. Non pas, alors, que dans cette conception le texte d'arrivée soit destiné à effacer le texte de départ, à le remplacer, comme le voudrait le discours traductologique uniquement sensible à la réception de la traduction, mais qu'au contraire les deux textes se placent dans un rapport d'égalité - un rapport fonctionnant dans les deux sens - où l'original soit toujours présent sous les espèces d'une mémoire à l'œuvre dans le texte traduit. ${ }^{1}$

\section{Le geste et la langue}

Si Kundera est cité ici, ce n'est pas que l'origine Mittel-Europa créerait automatiquement une parenté d'inspiration dont nombre de critiques à tendance journalistique abusent (après tout, Kundera n'est pas né à Prague!), mais parce qu'il y a dans les deux écritures un souci similaire qui est tout à fait frappant: le souci du geste. L'Immortalité, l'ouvrage de Kundera en question, est précisément fondé sur l'analyse d'un geste qui crée un, puis des personnages, et des histoires, enchaînant des variations sur la perception de ce geste, mode de composition qui était déjà d'ailleurs repérable dans l'Insoutenable légereté de l'être (1984) avec, par exemple, le leitmotiv du chapeau melon.

Chez Kafka, la présence du geste, l'affirmation d'un message et d'un langage du corps sont constantes, d'autant plus frappantes que ce gestuel semble se décrire et se déployer sans qu'il soit pourtant productif d'images, j'entends d'images objectives, réalistes, précises,

1. Voir sur cette problématique, qui est celle d'une approche herméneutique de la traduction, A. Nouss, «Translation: Art or Science? A Hermeneutic Reading", Proceedings of the Nth Scandinavian Symposium on Translation Theory (University of Turku, 1992) et «Une traduction n'est pas une application», Meta (à paraître, 1993). 
cinématographiques en ce qu'elles s'inscriraient dans une écriture dynamique et une continuité narrative. Les gestes chez Kafka sont davantage théâtraux, tendant vers l'exagération dramatique, l'expressionnisme. C'est de ce côté que le tire Adorno: «Il faut avoir lu le Verdict, la Métamorphose, le Chauffeur, dans les brochures noires que Kurt Wolff éditait dans sa collection Der Jüngste Tag pour situer Kafka dans son horizon authentique: l'expressionnisme. (...) Kafka sauve l'idée de l'expressionnisme en appliquant à la littérature l'effet de la peinture expressionniste, au lieu de guetter vainement l'écho des mots primitifs.» (Adorno, 1986, pp. 231 et 234) Walter Benjamin lisait déjà Kafka dans les mêmes termes: «Comme le Greco, derrière chaque geste, Kafka déchire le ciel; mais, comme chez le Greco - patron des expressionnistes -, le geste demeure l'élément décisif, le centre même de l'action.» (Benjamin, 1983, p. 202)

Or l'expression d'une telle gestualité est sans doute facilitée par la nature et la structure de la langue allemande. Il suffit de considérer le jeu des préfixes et des formes verbales pour percevoir cette prégnance sémantique. Georges-Arthur Goldschmidt écrit dans son Quand Freud voit la mer: «En allemand, tout part du corps, tout y revient, tout le traverse: der Leib (le corps qui est la vie même (sic)) a la même origine que das Leben (la vie), (...) der Leib est un tout autre corps que le Körper, le corps tel qu'il est issu du latin, et qui exprime le corps fonctionnel et aussi le corps de métier.» $(1988$, p. 16) Je renvoie encore à ses considérations sur la spatialité et la concrétude de l'allemand qui se forme «à partir de l'emplacement et du mouvement dans l'espace» (ibid., p. 17).

En matière de genre littéraire, ce qui fait la parabole ou l'apologue souvent, qu'on le prenne dans la tradition occidentale ou orientale, dans le hassidisme, le soufisme ou le zen, ce n'est pas tant sa teneur morale que son énonciation gestuelle, que sa langue qui tend vers le non-verbal, qui semble vouloir fuir sa lourdeur langagière. Benjamin rapprochait le geste et la parabole dans leur capacité commune à produire des commentaires (1983, p. 202). C'est le cas dans Vor dem Gesetz («Devant la Loi») comme partout ailleurs dans les récits-paraboles de Kafka et c'est davantage cette caractéristique qui tire l'œuvre de Kafka vers les littératures dites de spiritualité qu'un hypothétique contenu commun. 
Nous sommes ici au cœur d'un problème de traduction bien spécifique: le geste est en effet l'expression propre d'une personnalité, sa marque, son style, pourrait-on dire, et en même temps il n'appartient à personne, ni sa propriété ni sa création, comme le dit Kundera: «Car on ne peut considérer un geste ni comme la propriété d'un individu, ni comme sa création (nul n'étant en mesure de créer un geste propre, entièrement original et n'appartenant qu'à soi), ni même comme son instrument; le contraire est vrai: ce sont les gestes qui se servent de nous; nous sommes leurs instruments, leurs marionnettes, leurs incarnations.» $(1990$, p. 18)

C'est précisément l'individualité absolue du geste qui pour Adorno indique chez Kafka, comme dans l'expressionnisme, l'aliénation et l'enfermement du sujet moderne qui n'a plus le langage, facteur d'appartenance communautaire, pour exprimer sa vérité. Mais, comme je l'indiquais plus haut, le geste est aussi ce qui est exagéré, figé, grossi; il fait signe en ce sens vers l'inhumain, la chose, l'archaïque et s'accommode donc du silence, plus encore: il le réclame. Le geste, avant la langue: tel, il lui est à la fois inférieur, en tant qu'infra-humain, et supérieur, en tant qu'individualisé. Reste la question: comment faire passer d'une langue à une autre ce qui échappe à la langue, ce qui ne se saisit pas dans le langage? Car, si l'on peut dire que la «transposition de l'image gestuelle» ${ }^{2}$ est aisée car elle se passe de paroles, il n'empêche que cet aspect la rend alors entièrement dépendante du contexte pour sa signification (comme le geste corporel, au demeurant, tributaire de son encadrement socio-sémantique) et que cette indispensable contextualisation est à l'évidence un problème majeur pour le passage traductif: un contexte est encore plus difficile à rendre qu'un texte. Loin de n'être qu'un problème de traduction parmi d'autres, cette difficulté me semble illustrer la complexité même de toute pensée du traduire, car, lorsque je pose à propos du geste la question «comment faire passer d'une langue à une autre ce qui échappe à la langue», je ne fais que répéter en la particularisant l'aporie que Derrida a énoncée en une formule souvent citée: «(...) un corps verbal ne se laisse pas traduire ou transporter dans une autre langue. Il est cela même que la traduction

2. Selon l'expression de Monique Moser-Verrey, à qui je dois une lecture attentive et généreuse et dont les remarques m'ont été cruciales dans la rédaction finale de cet article. 
laisse tomber. Laisser tomber le corps, telle est même l'énergie essentielle de la traduction.» $(1979$, p. 312$)$ Or il nous est loisible d'interroger dans cette perspective l'œuvre de Kafka.

\section{Fragment et œuvre}

De ses textes-paraboles écrits de mots et de gestes, il en est un à valeur exemplaire, presqu'un jeu d'ombres, un théâtre de marionnettes, Vor dem Gesetz, «Devant la Loi» (souvent cité dans une lecture herméneutique ou philosophique) ${ }^{3}$. Le premier aspect qui nous retiendra est son double statut. Le texte figure dans l'avant-dernier chapitre du Proces, il précède l'exécution de Joseph K., il peut être considéré comme le noyau symbolique et signifiant du roman. On sait que celui-ci a été d'ailleurs bâti à partir de ce texte que Kafka dans son Journal baptise la «Légende» (tr. M. Robert, 1954, p. 410). Mais le texte a aussi été publié par Kafka (alors que la publication du Procès est posthume) en tant que tel, deux fois dans des périodiques puis dans le recueil intitulé Ein Landarzt paru en 1920.

Cela pose problème puisque, dans la version du Procès, le texte est étroitement encadré, il se présente dans une continuité, il est introduit par l'ecclésiastique dans la cathédrale qui va le donner à Joseph K. comme illustration de son discours et il est suivi par un échange de commentaires et d'interprétations divergentes entre les deux personnages sur le sens du récit. Il y a donc une tension manifeste entre l'insertion dans une continuité romanesque et l'isolement dans un recueil. Le texte tel qu'il nous parvient dans sa version double offre donc une dimension d'autonomie en même temps qu'il appelle une lecture intertextuelle.

Une telle tension n'est pas inconnue de la réflexion traductologique. On traduit toujours un texte dans sa cohérence et sa complétude; mais il n'existe pas ainsi, il existe au sein d'une œuvre, dans une littérature et dans une histoire de la langue. Steiner a souligné cette

3. Faut-il en rappeler le scénario? Un «homme de la campagne» arrive devant la porte de la Loi, ouverte mais barrée par un gardien qui ne le laisse pas entrer. Il restera là toute sa vie, le gardien lui confiant, juste avant qu'il ne meure, que cette porte lui était destinée à lui seul. 
appartenance et étudié les liens entre les écritures littéraire et traductive dans son Après Babel (1978). Et le traducteur doit voir son travail constamment irrigué de cette double conscience, le texte comme œuvre, le texte comme fragment.

Les traducteurs de Kafka en français diffèrent sur ce point dans leur approche. Vialatte, le pionnier, écrit dans une introduction ces lignes superbes:

On ne devrait pas parler de Kafka. Il y a trente ans que je le traduis, que je me suis fait son prophète et son cheval, sa nourrice et son homme de peine, et son lierre et sa mauvaise herbe, il y a trente ans que je ne veux pas le connaître. Quand une poule pond des œufs d'or, on ne va pas lui ouvrir le ventre. Qu'on me comprenne: on a eu cette chance inouile de trouver tout à coup dans la littérature une œuvre unique qui ne ressemblait à rien de connu, plus étrange que celle de Poe, plus lunaire que celle de Rimbaud, et d'ignorer à peu près tout de sa genèse, un ouf d'ange pour ainsi dire. Il n'y avait rien de plus pressé, me semble-t-il, que de ne pas en savoir davantage! Rien de plus urgent que d'ignorer! Ou de répandre de faux bruits! On restait libre d'inventer. C'était splendide. (1968, p. 31)

«On restait libre d'inventer.» Les pieux critiques kafkalogues ne se sont pas privés d'en faire le reproche à Vialatte. Toujours est-il que, pour Vialatte, il s'agit d'un corps-à-corps avec une œuvre nouvelle, absolument nouvelle, sans histoire. Il est vrai qu'il était le premier.

Bernard Lortholary, lui, s'inscrit délibérément dans l'histoire des traductions de Kafka. Curieusement, il semble parfaitement illustrer la conclusion du texte de Jacques Derrida que nous évoquerons plus loin, qui pose à partir de Devant la Loi le rapport de l'historicité de la loi et de l'historicité de la littérature, qui fait de la littérature un lieu privilégié d'observation et d'analyse de la loi. Lortholary commence ainsi sa «Note sur la traduction» du Proces : «Pourquoi retraduire le Proces? Pourquoi refaire le travail d'Alexandre Vialatte, publié en 1933 et sans cesse réédité pendant quarante ans, puis judicieusement révisé par Claude David en 1976?» (1983, p. 23) Et il répond en citant les 
«lois régissant la propriété littéraire». Il est vrai qu'en 1983 apparaissent deux nouvelles traductions parce que plus de cinquante ans s'étaient écoulés depuis la première parution en français. «On pouvait donc retraduire le Proces; pourquoi devait-on le faire?» (Ibid.) continue-t-il et les trois raisons qu'il invoque ont également à voir avec l'histoire des traductions du texte. Il cite d'abord le vieillissement inévitable des traductions dû à l'emploi par le traducteur de tournures «de son époque» pour pallier les pertes du passage d'une langue à l'autre. Puis il évoque les arguties juridiques qui ont empêché la traduction révisée de Claude David d'être correctement «lisible» dans l'édition de La Pléiade. Enfin, il s'attache à la coloration stylistique de la traduction de Vialatte, trop éloignée selon lui de l'humour noir et du "phrasé limpide et dur» (ibid., p. 24) de Kafka.

Georges-Arthur Goldschmidt, enfin, écrit lui aussi sa «Note sur la traduction». Mais lui ne traite - et ainsi commence-t-il sa note que du «texte de Kafka». Plus encore, il semble délibérément sauter l'histoire des traductions: «Toute traduction retraduite doit être aussi proche que faire se peut de l'original.» (1983, p. 28) Et il enchaîne sur des considérations concernant la gageure impossible de la double fidélité (aux langues de départ et d'arrivée) exigée du traducteur pour conclure: «Le texte doit, si on veut, peser le même poids dans l'une et l'autre langue; être donc à la fois exact et dans le ton. Venu de la littérature de départ, il doit aboutir à la littérature d'arrivée, s'y intégrer à sa manière, sans rien perdre de ce qu'il est.» (Ibid., p. 29) Il rend cependant compte de la première traduction de Kafka mais presque comme un effet de littérature dont sa propre démarche traductrice est indépendante: «Il faut rendre hommage à Alexandre Vialatte, confronté pour la première fois à un tel texte. C'est à lui que le public français dut de connaitre et de comprendre ce livre.» (Ibid., p. 29)

Si différentes positions se font donc entendre quant à la traduction de Kafka, c'est sans doute que le texte de Kafka appelle la traduction comme d'autres (Adorno) ont dit qu'il appelle l'interprétation. À cet appel, on peut répondre directement ou au contraire répondre dans l'histoire de cet appel. La traduction ne diffère pas ici de l'interprétation et Gadamer (1976, pp. 94-99) a montré qu'il existe deux pôles herméneutiques, figurés emblématiquement par Schleiermacher et Hegel: l'un qui tend à une restauration extérieure, se voulant objective, 
du cadre historique du texte interprété, l'autre visant à une intégration intérieure par la médiation avec le présent du sujet interprétant. La première compréhension se veut en dehors de son histoire, sinon de l'histoire; la seconde, au contraire, trouve son lieu dans le flot de l'histoire. Il est aisé de comprendre que, face à la fuyante intelligibilité du texte de Kafka, les traducteurs choisissent l'une ou l'autre option. ${ }^{4}$

On sait que Derrida, dans une conférence intitulée «Devant la loi» (avec minuscule), fait de ce double statut du texte de Kafka, de cette double identité de fragment et d'œuvre, l'articulation prouvant que ce qui fait l'œuvre en littérature, ce n'est ni le contenu ni la forme mais «les mouvements de cadrage et de référentialité» $(1984$, p. 186). Or cette limitation ne peut s'opérer que sous la garantie d'une loi supérieure à celle inhérente à l'écriture: le code socio-historique qui fixe les normes d'un texte, ses droits de propriété et qui lui attribue ou non un auteur. Mais Derrida montre que ce texte-ci, posant l'impossibilité de connaître la loi, suggère que la littérature, dans le même temps qu'elle affirme la loi, peut de par sa nature la nier, la contourner: il est le récit, soumis à la loi, de l'interdit qui pèse sur la loi et qui la rend impossible à atteindre: «(...) le récit devient le récit impossible de l'impossible. Le récit de l'interdit est un récit interdit». (Ibid., p. 181) Car il ne se passe rien dans ce récit, qu'une attente - que Derrida identifie comme «differance» (ibid., p. 183) - proprement énigmatique, au sens étymologique: l'homme de la campagne reste jusqu'à sa mort sans y pénétrer devant la porte menant à la loi et qui lui était pourtant destinée, à laquelle il avait droit. Le lecteur est devant un récit qui ne raconte rien et dont il ne peut interpréter le contenu, renvoyé qu'il est à la multitude des significations possibles. La fable ici n'a pas de morale sinon celle de son énonciation. Malaise qu'au demeurant la critique a suffisamment cerné comme aspect essentiel de l'écriture de Kafka. Or une telle dynamique du texte, le déplacement constant qu'ont décrit Deleuze et Guattari (1975), se retrouve en traductologie dans l'analyse de la réception: le texte d'arrivée ne dit rien de plus que n'a dit le texte de départ sinon la discursivité de son énonciation, née de son historicité. Derrida présente d'ailleurs, de manière pertinente pour notre propos,

4. Sur l'insaisissabilité du texte de Kafka, je renvoie aux ouvrages de Marthe Robert (1979), Maurice Blanchot (1981) et Régine Robin (1989). 
Vor dem Gesetz comme un«original»: «Malgré la non-identité à soi de son sens ou de sa destination, malgré son illisibilité essentielle, sa «forme» se présente et se performe comme une sorte d'identité personnelle ayant droit au respect absolu. (...) Une mauvaise traduction sera toujours appelée à comparaître devant la version dite originale qui fait référence (...)» (1984, p. 185). Intégrité qui donne à lire l'inintégrable et qu'il est possible de repérer dans le syntagme-titre dont l'importance est indiquée par sa reprise en incipit.

\section{Entre Vor et Gesetz}

Entre Vor et Gesetz, il y a tension puisque Vor implique une notion de décalage, quelle que soit sa définition, il annonce une relation vers quelque chose et donc un mouvement, alors que Gesetz implique, de par sa racine sémantique, une staticité, une pesanteur, une gravité (aux deux sens du mot en français puisque gesetzt, l'adjectif, signifie posé, sérieux, grave).

Gesetz désigne d'une manière générale la loi, le principe, le précepte, aux sens moral, juridique, philosophique. Mais on constate dans ses acceptions un penchant sémantique vers la loi en tant que loi écrite, dans le domaine juridique (le code) et dans le domaine religieux (la Loi de Moïse, l'Ancien Testament ou encore le Pentateuque: das Gesetz und die Propheten ou le Décalogue: Gesetztafeln; die Tafeln des Gesetzes), là où le français pourrait dire «l'Écriture». L'autorité inhérente à la tradition religieuse n'est-elle pas l'articulation qui permet une seconde dérive sémantique vers la notion de scripturalité? Le lexique typographique admet un gesetzt signifiant «sur le marbre» ${ }^{5}$ et un Gesetz ancien désigne dans le domaine littéraire la strophe ou le verset. ${ }^{6}$ Le titre pourrait ainsi se lire: «Devant l'écriture». Et peut-être ne doit-on pas oublier que si setzen couvre le champ sémantique du

5. Pons-Großwörterbuch (Stuttgart, Emst Klett Verlag, 1988), p. 250.

6. Jacob et Wilhelm Grimm, Deutsches Wörterbuch (München, Deutscher Taschenbuch Verlag, Band 5, 1984), p. 4075; Harrap's Standard German-English Dictionary (London, Harrap, Part I, Vol. 2), p. 74; Grand Dictionnaire allemand-français (Paris, Garnier, 1979), p. 176. 
placement et de l'établissement, il contient aussi la notion d'événementialité comme le prouve un es setzt familier au sens de «il y a»?. L'écrit, après tout, fixe aussi ce qui a eu lieu. Nous serions en pleine phénoménologie, approche qui ne me semble pas étrangère ni à la sensibilité philosophique de Kafka, ni à son attention aux formes langagières dialectales ou inusitées qui justifie au demeurant cette brève incursion philologique.

Ce n'est pas, en tous cas, la seule loi au sens juridique que vise le texte, muet d'ailleurs sur le domaine spécifique de cette loi, mais aussi la loi comme écriture. Comment peut-on en douter en relisant les lignes qui précèdent la parabole dans le Proces: «(...) il est dit de cette erreur dans les écrits qui précèdent la Loi: Une sentinelle (...)» (tr. A. Vialatte, 1968, p. 346). De même en se souvenant de ce passage rayé par Kafka et inclus en note dans la traduction de Goldschmidt (1983, p. 282): «Lorsqu'il eut dit cela il s'arrêta; il remarqua qu'il venait de parler et juger d'une légende; il ne connaissait même pas l'Écriture dont la légende était extraite et les explications lui étaient tout aussi inconnues.» Nous sommes donc en présence, avec cette légende, d'un écrit précédant l'écrit: l'écrit «Vor dem Gesetz» est lui-même, au sens temporel, Vor dem Gesetz. Récit d'avant le récit qui évoque aussi une réalité traductologique: le texte de départ se situe avant le texte d'arrivée et pourtant ne sera connu que par ce dernier.

Quant à Vor, il signifie: «devant», «avant», «contre», «en présence» et aussi «à cause de», bref toutes définitions qui impliquent le rapport comme en témoignent les mots composés intégrant ce préfixe: Vorbedacht, Vorbedingung, Vorhang, Vorkehrung, Vormittag... Le rapport n'est pas, comme en français, de séparation mais de continuité; et donc être devant la loi, c'est déjà peut-être ne pas l'atteindre mais d'une certaine manière en faire partie, y être soumis ${ }^{8}$, de même qu'une

7. Attesté dans le Duden (Mannheim, Wien, Zurich, Bibliographisches Institut, Band 5, 1980), p. 2387.

8. Ce que Derrida indique lorsqu'il remarque que c'est l'homme de la campagne lui-même, non le gardien, qui s'empêche de passer la porte (à lui destinée, de surcroît): «Il doit s'obliger lui-même, se donner l'ordre non pas d'obéir à la loi mais de ne pas accéder à la loi qui en 
question a partie liée avec la réponse - et l'exemple n'est pas innocent puisque la seule parole de l'homme de la campagne au gardien est une question -, de même qu'une herméneutique traductologique, comme nous l'indiquions en introduction, pose un lien dialectique entre textes de départ et d'arrivée.

Goldschmidt écrit: «Il y a en allemand une sorte d'Urwüchsigkeit, une sorte de croissance de la langue à partir d'elle-même, qui lui fait à chaque instant revivre, en quelque façon, son enfance linguistique. (...) C'est que l'allemand est, rappelons-le, une langue essentiellement populaire, tirant ses étymologies d'elle-même.» (1988, p. 22; p. 30) Transparence profonde qu'un Kafka, plus que quiconque, ne pouvait ignorer en écrivant. L'allemand de Kafka, on le sait, est cette langue qui vise au plus près de son noyau de signifiance, qui tend à s'y brûler pour en restituer la braise jamais éteinte de rayonnante froideur. Les critiques ont longuement décrit et analysé la neutralité et la sobriété de son écriture, si différente de la luxuriance baroque des autres auteurs germanophones de Prague. L'explication sociologique est souvent avancée à cet égard: Kafka collait à l'idiome pragois, langue d'une minorité linguistique, dont la séparation d'avec l'allemand vivant entrainait l'appauvrissement et la simplicité. Une autre perspective met en rapport cet isolement linguistique revendiqué par Kafka et la solitude qu'il expérimentait biographiquement et dont il se faisait un fondement philosophique. Mais une autre approche peut encore considérer la langue de Kafka comme une langue de traduction. Si en effet l'allemand était sa langue maternelle, les pages du Journal ou de sa correspondance nous informent qu'il la considérait comme une langue empruntée et même volée, selon ses propres termes. Dès lors sa fascination pour le tchèque, le yiddish et l'hébreu, les fantasmes qu'il nourrit à leur endroit pourraient nous inviter à voir son écriture comme une entreprise de traduction: son cuvre fonctionnerait comme un texte d'arrivée, bien particulier en ce que le traducteur serait devant ces langues: comme

somme lui fait dire ou lui laisse savoir: ne viens pas à moi, je t'ordonne de ne pas venir encore jusqu'à moi. C'est là et en cela que je suis la loi et que tu accéderas à ma demande. Sans accéder à moi.» (1984, p. 183)

9. Voir note 1. 
l'homme de la campagne, toute sa vie, il recevrait leur éclat sans pénétrer en elles. Ce statut ambigu correspondrait, au niveau langagier, à la lecture souvent faite du texte de Kafka reçu comme le commentaire d'un livre premier et fondateur mais absent.

\section{Avant de traduire}

Cynthia Ozyck, dans un très bel essai intitulé «A translator's monologue», articule ce qu'elle appelle la fausse mais nécessaire idée selon laquelle un texte n'est jamais traduit mais plutôt mis au jour, découvert, dévoilé (uncovered) (1989, p. 200). Le nouveau texte est en attente dans la nouvelle langue, il suffit de l'y trouver. Aussitôt s'impose l'image de la lumière irradiant de derrière la porte, la porte de la langue donc ou la porte de l'écrit, Gesetz. Avec cette précision qu'on ne franchit jamais la porte, qu'une traduction n'est jamais définitive, qu'elle n'est qu'une transgression. Et la tension lexicale et sémantique entre Vor et Gesetz nous apprendrait que, dans traduire comme dans écrire, le processus est essentiellement dynamique, tous les éléments sont liés (émission/réception, avant/après...). Traduire comme écrire, c'est se situer dans l'entre-deux. Entre-deux qui représentait pour Walter Benjamin l'idéal de la traduction puisque pour lui toutes les langues ensemble, révélées les unes aux autres par la traduction, formaient la langue pure (1971, p. 267), la «lalangue» dirait Lacan. Remarquons que dans son essai sur Kafka (1983), Benjamin parle aussi d'«entre-monde» pour désigner celui de Kafka. ${ }^{10}$

10. Un mot, à cet égard, sur l'interprétation de «l'homme de la campagnex. On a pris l'habitude de voir dans cet homme de la campagne la projection et la personnification d'une expression hébraique passée dans le yiddish. Le am haaretz, «peuple de la terre» au sens littéral, désigne dans la littérature talmudique puis dans l'expression populaire yiddish les individus illétrés qui n'ont qu'un rapport obéissant et stupide avec la loi. Heinz Politzer (1962) et Régine Robin (1989) rapportent cette origine. Je ne suis pas si sûr de cette étymologie. S'il est vrai que l'expression a été notée par Kafka dans ses cahiers, son sens traditionnel ne me semble pas parfaitement concorder avec le profil de l'homme qui demande à entrer dans la loi. Ne serait-ce que ce désir le qualifie déjà positivement et cette requête pourrait être celle des plus grands sages. Il est au contraire occupé, presque obsédé, par la loi et finalement il y sacrifie sa vie. D'autre 
Nous pourrions donc traduire Vor dem Gesetz par «Avant le traduit» ou "Avant l'écrit» ou encore «Avant le récit». Nous mentionnions plus haut que Derrida avançait que, parce qu'il y est question de l'inaccessibilité de la loi, le récit lui aussi ne raconte rien, est inaccessible, impossible. L'accès à la loi, parce que c'est la loi, est toujours repoussé, différé. Derrida écrit (1984, p. 184): «Représenté par le gardien, le discours de la loi ne dit pas «non» mais «pas encore», indéfiniment. D'où l'engagement dans un récit à la fois parfaitement fini et brutalement interrompu, on pourrait dire primitivement interrompu.» Or le texte et la réflexion de Derrida semblent aussi être interrompus. En effet, alors qu'il situe lui-même son intervention dans une particularité traductive (il lit une traduction en anglais du texte qu'il a écrit en français sur le texte allemand de Kafka), il n'opère pas le passage conceptuel vers un autre impossible, un autre interdit: la traduction. On n'arriverait jamais vraiment devant la loi comme on n'arriverait jamais vraiment à traduire. Derrida lui-même pourtant cite la traduction comme un des phénomènes auxquels s'attache la juridiction littéraire - et nous faisions précédemment allusion à la loi qui permit les nouvelles traductions de Kafka.

Comment ne pas rapprocher Gesetz et Übersetzung, la traduction, tous deux formés de setzen? Derrida, dans les interventions recueillies sous le titre de l'Oreille de l'autre, place au demeurant la traduction sous le signe de la loi puisqu'à la suite de Benjamin il lit dans le texte premier un appel, une demande: «Car si la traduction est endettée à l'égard de l'original (c'est cela la tâche, la dette (Aufgabe)), c'est que déjà l'original est endetté à l'égard d'une traduction à venir. Ce qui fait que la traduction, c'est aussi bien la loi.» (1982, p. 202) Loi en tant que ce qui impose le désir, ce qui fait que l'homme de la

part, je ne peux m'empêcher de rapprocher ce Mann vom Lande du Landartz, le médecin de campagne, qui donne son titre au recueil où se trouve également «Vor dem Gesetz». Cette campagne ne serait-elle pas plutôt l'univers indifférencié, illimité, sans frontières entre la nuit et le jour, la vie et la mort, entre-deux que la loi a précisément pour rôle d'ordonner et d'où Kafka souffre de ne pouvoir s'arracher, sinon par l'écriture? L'homme de la campagne, s'il ne sait pas lire ou s'il n'y parvient pas, comme le dit Derrida, a du moins la perception de la Loi. 
campagne accepte de rester là des années, toute sa vie, dans l'attente d'entrer. Vor dem Gesetz illustrerait parfaitement ce propos de Derrida (faut-il voir un effet de la «differance» que ce lien que je dessine à sa place?). Übersetzen, littéralement «poser au-delà», indiquerait bien que l'acte du traduire implique un franchissement, plus que le passage compris dans le français «tra-duction», et reconnaît donc l'existence d'un seuil, d'un interdit, d'une porte. Vor dem Gesetz devient récit métaphorique de l'aporie fondatrice ${ }^{11}$ qui revient pour relayer une phénoménologie se heurtant aux limites de sa méthode: la traduction est possible et impossible. Là où vor (dem Gesetz) pose l'impossible, über(-setzen) énonce le possible. Entre vor et über se dessine aussi un rapport de temporalité dont participe l'essence de la traduction: si elle travaille sur un matériau langagier, elle travaille tout autant sur une trace historique, celle qui relie l'avant du texte de départ à l'après du texte d'arrivée. Mais le parcours de l'un à l'autre n'est pas une progression dont le terme marquera l'oubli de la genèse. Le mythe de l'occultation de l'original a fait long feu ${ }^{12}$ : le processus traductif revèle une temporalité spécifique où ce qui vient après donne corps et existence à ce qui est venu avant mais où pourtant il ne serait pas venu après s'il n'y avait eu de l'avant. ${ }^{13} \mathrm{La}$ traduction est constituée de ce rapport: la porte est là pour l'homme de la campagne, certes, mais elle n'est pas fermée et laisse passer la lumière, comme une métaphore de la temporalité ininterrompue constituant l'être de la traduction.

C'est bien en effet une histoire de traduction ou d'interprétation. «C'est sur la justice que tu te méprends» (tr. A. Vialatte, 1968, p. 346; Lortholary et Goldschmidt préfèrent «le tribunal»), dit l'ecclé-

11. Que l'on a pris l'habitude de définir comme «objection préjudicielle» dans le discours traductologique (voir J.-R. Ladmiral, 1979, p. 85 et suiv.).

12. Voir, sur le plan sémiologique, B. Folkart (1991) et, sur le plan philosophique, H. Meschonnic (1973a).

13. Voir, pour une considération herméneutique de cette problématique, A. Nouss, «Herméneutique et traduction: le franchissement des distances», Actes du Congrès international de traductologie (Université de Vienne, septembre 1992; à paraître). 
siastique à Joseph K. pour introduire la parabole et d'autre part, quand il lui raconte la parabole, il la traduit en fait puisque, comme nous l'avons vu, elle existe avant la loi, avant l'écrit, avant le verset, avant le connu. Vor dem Gesetz en tant que récit est également Vor dem Gesetz, avant la parole, celle que Joseph K. reçoit de son interlocuteur, logos ordonnateur du sens et du réel, et, dans un horizon traductologique, avant le texte d'arrivée, qui, de toutes les versions possibles, aura choisi d'en arrêter une: quitter, pour l'homme ${ }^{14}$-traducteur, la campagne illimitée des compréhensions de l'original pour pénétrer dans l'espace restreint du sens fixé qui s'attachera à sa traduction. ${ }^{15}$

\section{Des noms}

La parole est aussi ce qui nomme. Dans la légende «Devant la Loi», le récit d'avant le récit, l'homme de la campagne n'a pas de nom. Il fait partie dans la série des personnages de Kafka de ceux dont l'identité n'a même plus le secours d'une initiale. Il n'a pas de nom et pourtant n'est pas anonyme. Le gardien sait qui il est et lui confie qu'il n'est pas n'importe qui puisque la porte de la loi lui était destinée.

Dans une autre réflexion sur la traduction, Derrida met celle-ci en rapport avec la question du nom propre. Entre le texte original et sa traduction, on peut déceler la même tension qu'entre le nom propre et le nom commun, qui est celle qui existe entre l'affirmation du particularisme et l'exigence de l'universel. Un texte dit sa vérité qui est vraie si elle lui est propre. Comment en même temps l'exprimer à l'autre pour que l'autre la comprenne, la prenne avec, avec lui, la mêle à sa vérité à lui? «Tout nom propre est travaillé par ce désir: traduis-moi, ne me traduis pas. Ne me traduis pas, c'est-à-dire respecte-moi comme nom propre, respecte ma loi de nom propre qui est au-dessus de toutes langues et traduis-moi, c'est-à-dire comprends-moi, conserve-moi en

14. Je n'utilise ce terme qu'en référence au texte de Kafka.

15. Remarquons, pour filer la métaphore, que le parcours traductif ne s'arrête pas au passage de la première porte, celle devant laquelle se tient le gardien. Le texte nous dit que la porte mène à une enfilade de salles dont il n'est pas précisé le nombre. De même, une traduction est ouverte à l'histoire de toutes les versions ultérieures. 
langue universelle, suis ma loi, etc.» $(1982$, p. 137) Derrida fait même de ce double bind, «traduis-moi, ne me traduis pas», la loi de toute traduction, à partir du mythique épisode fondateur de la Tour de Babel. $^{16}$

L'homme de la campagne est soumis à cette ambivalente contrainte. Il refuse le dévoilement de son nom propre, nom secret, par peur qu'il devienne traduit, c'est-à-dire commun (aux deux sens), mais en même temps, il aspire au Gesetz, à la loi qui va lui donner un nom propre connu, c'est-à-dire un nom propre (l'entrée lui est réservée) et commun (tous ne veulent-ils pas y entrer?).

Reste le problème du rapport entre ce nom premier, originel, nom intérieur en quelque sorte, et ce nom second qui doit être reçu de l'extérieur. «Mais ce qu'on peut se demander, pose Derrida, c'est si, sous le nom propre ou les noms propres publics et connus en quelque sorte, qui peuvent être les noms patronymiques ou d'autres, il n'existe pas un nom propre inconscient ou secret, à la recherche duquel nous sommes, ou bien le lecteur ou l'analyste doit être.» (1982, p. 140) Cette tension, c'est le «traduis-moi, ne me traduis pas». Curieusement, Derrida avance que ce nom secret pourrait être non pas forcément de l'ordre du verbal mais aussi du gestuel ou de l'olfactif (1982, p. 141). Nous avions déjà approché plus haut le geste comme ce qui appartient à la fois à un vocabulaire commun et à un lexique individualisé: nous avons tous les mêmes gestes, héritiers d'une même disposition corporelle, mais nous les personnalisons d'une indissociable spécificité. Un geste, comme un rire, se reconnaît entre mille. Le geste se trouve ainsi des deux côtés de la porte interdite qui sépare le traduisible de l'intraduisible, l'en-deçà de l'au-delà du langage. «Traduis-moi»: passe la porte; «ne me traduis pas»: reste devant, dit la Loi; et la traduction devient le passage possible-impossible qui définit la Loi et qui en reçoit en retour à la fois son essence et son fonctionnement.

16. Voir, sur ce point, son commentaire de «la Tâche du traducteur» de W. Benjamin, «Des Tours de Babel» (Psyché. Inventions de l'autre. Paris, Galilée, 1987) et A. Nouss, «Texte et traduction: du sacré chez Jacques Derrida» (Religiologiques, 5, Montréal, 1992). 
Or Derrida ne croit pas à un nom absolument propre, absolument pur, car s'il peut être énoncé dans un système, dans un réseau, si quelqu'un d'autre peut m'interpeller, alors ce nom risque d'être contaminé et perdra de son secret, de son absolu: $\ll S i$ le nom propre le plus secret n'a son effet de nom propre que dans la mesure où il risque la contamination, donc la déviation à l'intérieur d'un système de relations, à ce moment-là, l'adresse pure est impossible. Je ne peux jamais être sûr que quand quelqu'un me dit, te dit, me dit «toi», «toi» ça ne puisse pas être un «toi» quelconque. Je ne peux jamais être sûr que l'adresse secrète ne puisse pas être détournée, comme tout message, comme toute lettre, pour ne pas arriver à destination.» (1982, p. 143) Derrida, significativement, conjugue la loi de la traduction sous le signe d'une parole adressée à une deuxième personne grammaticale, proche ici de l'ontologie de Martin Buber qui plaça son projet de traduction biblique entrepris avec Franz Rosenzweig à la lumière de sa philosophie du «Ich und Du».

Un «toi» qui pourrait être n'importe quel «toi», n'est-ce pas l'angoisse même de l'homme de la campagne qui l'empêche d'entrer et qui lui fait formuler sa question: "comment se fait-il que pendant toutes ces années personne à part moi n'ait demandé à entrer?» (Goldschmidt, 1983, p. 243)? Et lorsque Derrida donne l'exemple de la lettre qui risque de ne pas parvenir s'impose le souvenir de cet autre extraordinaire parabole de Kafka, le «Message impérial», qui, après le récit du message jamais parvenu car disparu dans les contingences du trajet, se conclut par l'apostrophe: «Mais toi, tu es assis à ta fenêtre, et dans ton rêve tu appelles le messager quand vient le soir.» (Vialatte, 1969, p. 143). «Toi»: la plus directe des adresses et la plus indécise.

Et nous pouvons alors comprendre le choix privilégié de cette forme textuelle par Kafka. La parabole s'énonce comme un récit pur, une pure narrativité, sans géographie, histoire ou psychologie. Cette pureté-là assure son ouverture sémantique: elle est à la fois «forme du vivre» et distante par rapport au rél comme l'indique Meschonnic (1973b, pp. 111-115). Comme le geste et comme la porte de la loi, elle appartient à tous et à quelqu'un en particulier, si bien que chacun peut s'y reconnaître. Elle serait donc, sur le modèle du nom propre, à la fois un appel désespéré à la traduction et l'interdit de cette traduction 
comme impossible, le Gesetz comme écrit («traduis-moi») et le Gesetz comme loi («ne me traduis pas»).

\section{Du sens et de ses clés}

Nous avons précédemment évoqué le double statut de «Devant la Loi», cuvre et fragment. Sans doute devons-nous dire maintenant que c'est la nature même de l'écriture de Kafka (dans sa réalité et dans son projet) que de permettre cette ambivalence. Répétons, après tant d'autres, que le sens n'y est jamais figé, qu'il circule, qu'il est autant dans l'énonciation que dans la réception, qu'il est dans leur dialogue. Le premier sens de la parabole que les multiples interprétations n'arrivent à cerner, c'est de montrer, non pas dire (la pensée de Wittgenstein peut être éclairante ici), que le sens, précisément, ne peut être cerné. Le sens circule, toujours. On peut et on doit lire le texte de Kafka à la fois dans une continuité jamais achevée et dans une fragmentation autarcique. Le texte de Kafka est toujours avant ou après, est toujours en fuite, toujours devant, Vor dem Gesetz. «Hyper-signe, le texte kafkaïen est une méta-fiction qui dénie à l'avance toute interprétation, qui met perpétuellement en abîme le premier texte absent sur lequel le légendaire, le tenant-lieu viendra se greffer», explique justement $R$. Robin (1989, p. 20). En cela le texte de Kafka est emblématique du processus de traduction.

Traduire, c'est se placer dans le risque de ce double axe, paradigmatique et syntagmatique, et le représenter (au sens de répétition et de la Darstellung allemande) comme Kafka l'a fait avec ce texte au double statut.

Et il n'est pas vain que ce soit dans le Procès et dans Devant la Loi: la loi, comme la langue demande à être interprétée, n'existe qu'interprétée, que traduite et plus l'écriture ira vers une loi et une langue épurées, ce qui est suprêmement le cas dans Devant la Loi, plus le processus sera flagrant. La loi, au-delà des strictes interprétations théologiques, philosophiques ou politiques, semble chez Kafka ne pouvoir être perçue que dans cette perpétuelle mouvance ou plutôt trouve son être dans cette perpétuelle mouvance, à l'image de ce qui se donne à comprendre dans la littérature midrachique ou talmudique juive dont ce texte semble être extrait. En effet, les commentaires rabbiniques 
traditionnels concourent, au-delà de leurs visées prescriptives ou morales, à nous faire comprendre que le sens réside essentiellement et indéfiniment dans la seule quête du sens.

Jan Kott (1982), dans un essai sur Kafka, rappelle une parabole talmudique selon laquelle la Bible et tous les textes sacrés sont comme un château comportant des milliers et des milliers de chambres. Devant chaque chambre, une clé.

Mais la clé d'une autre chambre.

Et pour reprendre les propositions de Kundera sur l'authenticité citées en préambule: les textes, l'original et la traduction, sont comme des clés, non pas une serrure et une clé: texte de départ et texte d'arrivée, mais chacun une clé (ou encore un geste dont on n'est jamais sûr du sens, en absence de toute explication langagière). On n'en connaît pas la serrure et on ne sait même pas quelle porte elle ouvrira.

Université de Montréal

\section{Références}

\section{F. Kafka: les traductions}

DAVID, Claude (1980-1989). Euvres completes de Kafka. Paris, Gallimard, «La Pléiade».

GOLDSCHMIDT, Georges-Arthur (1983). Le Proces. Paris, Presses-Pocket.

LORTHOLARY, Bemard (1983). Le Procès. Paris, Flammarion, «GF».

ROBERT, Marthe (1954). Journal. Paris, Grasset. 
(1957). Préparatifs de noces a la campagne. Paris, Gallimard.

VIALATTE, Alexandre (1968). Le Procès. Paris, Gallimard, «Livre de Poche».

(1969). La Métamorphose. Paris, Gallimard, «Le Livre de Poche».

\section{Références générales}

ADORNO, Theodor W. (1986). «Réflexions sur Kafka». Prismes Critique de la culture et société (tr. par Geneviève et Rainer Rochlitz). Paris, Payot.

BENJAMIN, Walter (1983). «Franz Kafka». Essais, I (tr. par Maurice de Gandillac). Paris, Denoël/Gonthier, «Médiations».

(1971). «La Tâche du traducteur». Mythe et violence (tr. par Maurice de Gandillac). Paris, Denoel.

BLANCHOT, Maurice (1981). De Kafka d Kafka. Paris, Gallimard, «Idées».

DELEUZE, Gilles et GUATTARI, Félix (1975). Kafka - Pour une littérature mineure. Paris, Éditions de Minuit.

DERRIDA, Jacques (1979). «Freud et la scène de l'écriture», L'Écriture et la différence. Paris, Le Seuil («Points»).

(1982). L'Oreille de l'autre (Textes et débats avec Jacques Derrida, sous la direction de Claude Lévesque et Christie V. McDonald). Montréal, VLB éditeur.

(1984). «Devant la loi». Philosophy and Literature, éd. A. Phillips Griffiths. Cambridge University Press.

FOLKART, Barbara (1991). Le Conflit des énonciations. Traduction et discours rapporté. Montréal. Éd. Balzac. 
GADAMER, Hans-Georg (1976). Vérité et méthode (tr. par E. Sacre). Paris, Le Seuil.

GOLDSCHMIDT, Georges-Arthur (1988). Quand Freud voit la mer Freud et la langue allemande. Paris, Buchet/Chastel.

KOTT, Jan (1982). «A Cage in Search of a Bird». Partisan Review, vol. XLIX, $n^{\circ} 3$, pp. 340-350.

KUNDERA, Milan (1984). L'Insoutenable légèreté de l'être (tr. par François Kérel). Paris, Gallimard.

(1989). L'Immortalité (tr. par Eva Bloch). Paris, Gallimard.

LADMIRAL, Jean-René (1979). Traduire: théorèmes pour la traduction. Paris, Payot («Petite Bibliothèque Payot»).

MESCHONNIC, Henri (1973a). «Poétique de la traduction», Pour la poétique II. Paris, Gallimard.

(1973b). «La Parabole ou Kafka», Pour la poétique III. Paris, Gallimard.

OZICK, Cynthia (1989). «A Translator's Monologue». Metaphor and Memory. New York, Knopf.

POLITZER, Heinz (1962). Franz Kafka - Parabole and Paradox. Comell University Press.

ROBERT, Marthe (1979). Seul, comme Franz Kafka. Paris, CalmannLévy.

ROBIN, Régine (1989). Kafka. Paris, Belfond.

STEINER, George (1978). Après Babel. Une poétique du dire et de la traduction (tr. par L. Lotringer). Paris, Albin Michel. 